\title{
Biquad Gm-C Structures Which Use Double-Output Transconductors
}

\author{
Vladimir I. Prodanov \& Michael M. Green \\ Department of Electrical Engineering \\ State University of New York \\ Stony Brook, NY 11794-2350
}

\begin{abstract}
Two Gm-C biquad structures are derived. They use only two grounded capacitors and three transconductors, two of which have double-outputs. The realization of the double-output transconductors is discussed. It is shown that only two additional transistors are needed to convert a single-output transconductor to a double-output one suitable for implementation of the proposed structures. HSPICE simulations and experimental results of a 5-th order inverse Gaussian filter using the proposed biquad structures are given.
\end{abstract}

\section{INTRODUCTION}

In general there are two analog filter synthesis techniques: the passive prototype-based technique and the biquad-based technique. The latter is preferred when the filter transfer function has finite- $Q$ zeros.

In the last decade many different $\mathrm{Gm}-\mathrm{C}$ biquad structures for monolithic filter implementation have been reported [1], [2], [3]. They are based on a two-integrator loop. The transmission zeros are obtained by injecting weighted signals into the loop. As discussed in [2] there are two ways of achieving this without destroying the established two integrator loop poles:

1) By applying the input voltage to an appropriate node (or set of nodes). In many cases this procedure results in a structure which has floating capacitors and requires input buffering.

2) By feeding additional current (generated by an additional transconductor) into an impedance node. Using this approach one can design highly flexible biquads [3], [4] which use only grounded capacitors and does not require input buffering. Unfortunately, they use many (7-8) transconductance blocks, which implies high power dissipation and large chip area.

In this paper we present two biquad structures which employ only three transconductors and two grounded capacitors.

II. Derivation of the BIQUAD STRUCtures

Consider the simple structure shown in Fig. 1. The transfer functions $V_{1} / V_{i n}$ and $V_{2} / V_{i n}$ can be easily shown to be:

$$
\begin{aligned}
& \frac{V_{1}}{V_{i n}}=\frac{1+s \frac{C_{2}}{g_{m 2}}}{1+s \frac{C_{1}}{g_{m 1}}+s^{2} \frac{C_{1} C_{2}}{g_{m 1} g_{m 2}}} \\
& \frac{V_{2}}{V_{i n}}=\frac{1}{1+s \frac{C_{1}}{g_{m 1}}+s^{2} \frac{C_{1} C_{2}}{g_{m 1} g_{m 2}}}
\end{aligned}
$$

The poles of this circuit are thus given by:

$$
s=\frac{g_{m 2}}{2 C_{2}}\left(-1 \pm \sqrt{1-4 \frac{g_{m 1}}{g_{m 2}} \frac{C_{2}}{C_{1}}}\right)
$$

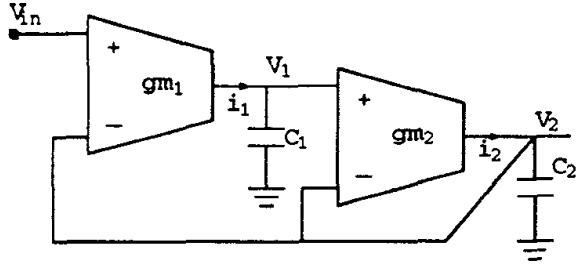

Figure 1: Gm-C lowpass structure
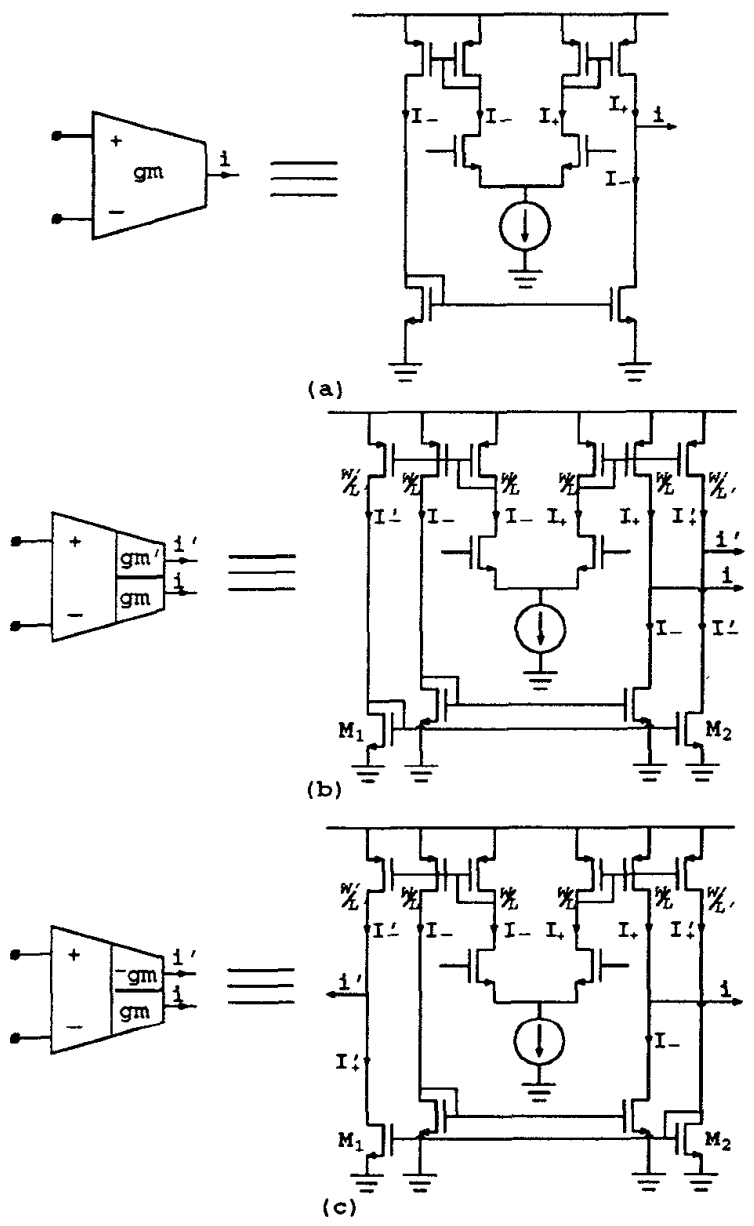

Figure 2: (a) Single-output transconductor; (b) and (c) Double-output transconductors

A pair of zeros is realized by adding an extra feedforward path; this can be done conveniently by simply adding an 
extra output to each transconductance block, as shown in Fig. 2. Notice that we can set the absolute value of the ratio $g_{m} / g_{m}^{\prime}$ by setting the appropriate $W / L$ ratios. The sign can be selected positive(negative) by choosing $M_{1}\left(M_{2}\right)$ to be diode connected as in Fig. 2(b)(Fig. 2(c)). The currents leaving these extra outputs can then be summed in an appropriate way. To perform this summation and convert the currents into a voltage, an additional single-output transconductance block is used as shown in Fig. 3.

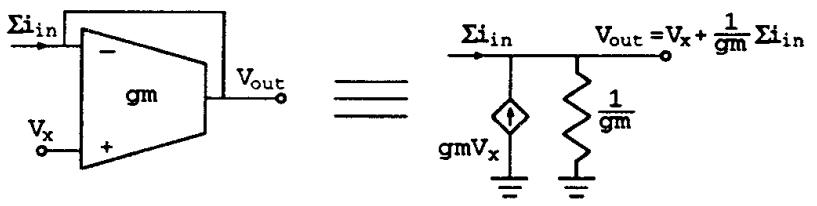

Figure 3: Using single-output transconductor as a summer: the circuit and its small signal equivalent

After making the above suggested modifications, the circuit in Fig. 4 results.

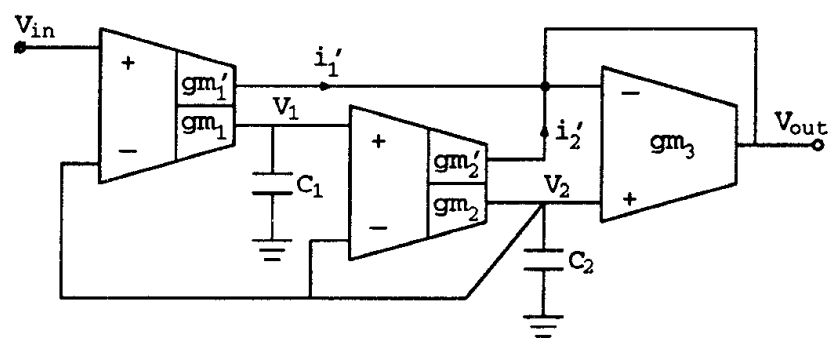

Figure 4: $\mathrm{Gm}-\mathrm{C}$ biquad structure

To derive its transfer function we write:

$$
V_{\text {out }}=V_{2}+\frac{1}{g_{m 3}}\left(i_{1}^{\prime}+i_{2}^{\prime}\right)
$$

or

$$
V_{\text {out }}=V_{2}+\frac{1}{g_{m 3}}\left[g_{m 1}^{\prime}\left(V_{i n}-V_{2}\right)+g_{m 2}^{\prime}\left(V_{1}-V_{2}\right)\right]
$$

Incorporating (1) and (2) into (5), we have the following filter transfer function:

$$
\frac{V_{\text {out }}}{V_{\text {in }}}=\frac{1+s\left(1+\frac{g_{m 1}}{g_{m 1}^{\prime}} \frac{g_{m 2}^{\prime}}{g_{m 2}} \frac{C_{2}}{C_{1}}\right) \frac{g_{m a 1}^{\prime}}{g_{m 1}} \frac{C_{1}}{g_{m 3}}+s^{2} \frac{g_{m 1}^{\prime}}{g_{m 1}} \frac{C_{1} C_{2}}{g_{m 3} g_{m 2}}}{1+s \frac{C_{1}}{g_{m 1}}+s^{2} \frac{C_{1} C_{2}}{g_{m 1} g_{m 2}}}
$$

From this transfer function we can derive the $\omega_{0}$ and $Q$ of the quadratic function in the numerator and the denominator:

For the denominator,

$$
\omega_{0 d}=\sqrt{\frac{g_{m 1} g_{m 2}}{C_{1} C_{2}}} \quad Q_{d}=\sqrt{\frac{g_{m 1}}{g_{m 2}} \frac{C_{2}}{C_{1}}}
$$

For the numerator,

$$
\omega_{0 n}=\sqrt{\frac{g_{m 1}}{g_{m 1}^{\prime}}} \sqrt{\frac{g_{m s} g_{m 2}}{C_{1} C_{2}}} \quad Q_{n}=\frac{\sqrt{\frac{g_{m 1}}{g_{m 1}}} \sqrt{\frac{g_{m s}}{g_{m 2}} \frac{C_{2}}{C_{1}}}}{1+\frac{g_{p 11}}{g_{m 1}^{\prime}} \frac{g_{m 2}^{\prime}}{g_{m 2}} \frac{C_{2}}{C_{1}}}
$$

By interchanging the identity of the input and output terminals as shown in Fig. 5 , it is very simple to show that (1), (2) and (5) will still hold, but with $V_{\text {in }}$ and $V_{\text {out }}$ interchanged. Thus the transfer function of the Fig. 5 circuit will be the reciprocal of the transfer function given in (6).

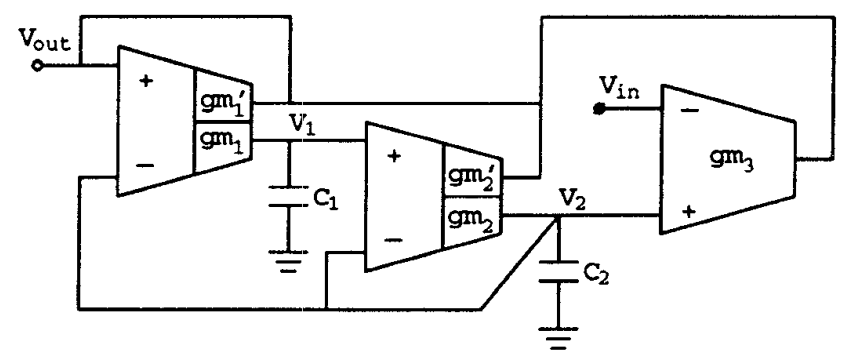

Figure 5: Gm-C biquad structure reciprocal to the one in Fig.3

Since in the last structure the initial two-integrator loop formed by $g_{m 1}, g_{m 2}, C_{1}$ and $C_{2}$ determines the zeros of its transfer function, one may break the feedback as shown in Fig. 6 without inflicting instability.
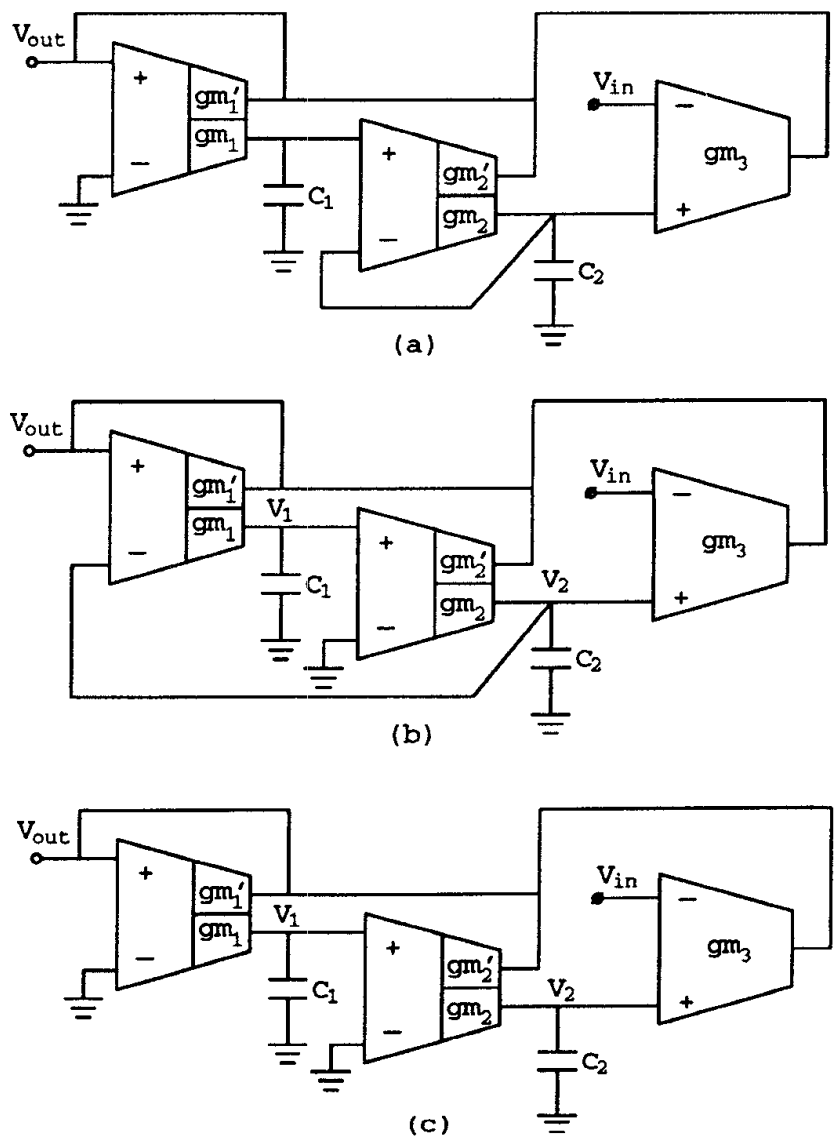

Figure 6: Additional structures derived by breaking a feedback loop in Fig. 5 circuit 
The resulting biquad configurations have the following respective transfer functions:

\section{Fig.6(a) Circuit}

$$
\frac{V_{o u t}}{V_{i n}}=\frac{s \frac{C_{1}}{g g_{1}}\left(1+s \frac{C_{2}}{g_{m 2}}\right)}{1+s\left[1+\frac{g m_{1}}{g m_{1}^{\prime}} \frac{g_{m 2}^{\prime}}{g_{m 2}} \frac{C_{2}}{C_{1}}\right] \frac{g m_{1}^{\prime}}{g m_{1}} \frac{C_{1}}{g m_{3}}+s^{2} \frac{g_{m 2}^{\prime}}{g_{m 2}} \frac{C_{1} C_{2}}{g_{m 3} g_{m 2}}}
$$

Fig.6(b) Circuit

$$
\begin{aligned}
\text { Notch } & \frac{V_{\text {out }}}{V_{\text {in }}} & =\frac{1+s^{2} \frac{C_{1} C_{2}}{g_{m 1} g_{m 2}}}{1+s \frac{g_{m 2}^{\prime}}{g_{m 2}} \frac{C_{2}}{g_{m 3}}+s^{2} \frac{g_{m 1}^{\prime}}{g_{m 1}} \frac{C_{1} C_{2}}{g_{m 2} g_{m 3}}} \\
\text { BP } & \frac{V_{1}}{V_{\text {in }}} & =\frac{s \frac{C_{2}}{g_{m 2}}}{1+s \frac{g_{m 2}^{\prime}}{g_{m 2}} \frac{C_{2}}{g_{m 3}}+s^{2} \frac{g_{m 1}^{\prime}}{g_{m 1}} \frac{C_{1} C_{2}}{g_{m 2} g_{m 3}}} \\
\text { LP } & \frac{V_{2}}{V_{i n}} & =\frac{1}{1+s \frac{g_{m 2}^{\prime}}{g_{m 2}} \frac{C_{2}}{g_{m 3}}+s^{2} \frac{g_{m 1}^{\prime}}{g_{m 1}} \frac{C_{1} C_{2}}{g_{m 2} g_{m 3}}}
\end{aligned}
$$

Fig.6(c) Cirquit

$$
\begin{aligned}
\mathrm{HP} & \frac{V_{\text {out }}}{V_{\text {in }}} & =\frac{s^{2} \frac{C_{1} C_{2}}{g_{m 1} g_{m 2}}}{1+s \frac{g_{m 2}^{\prime}}{g_{m 2}} \frac{C_{2}}{g_{m 3}}+s^{2} \frac{g_{m 1}^{\prime}}{g_{m 1}} \frac{C_{1} C_{2}}{g_{m 2} g_{m 3}}} \\
\mathrm{BP} & \frac{V_{1}}{V_{i n}} & =\frac{C_{2}}{1+s \frac{g_{m 2}^{\prime}}{g_{m 2}} \frac{C_{2}}{g_{m 3}}+s^{2} \frac{g_{m 1}^{\prime}}{g_{m 1}} \frac{C_{1} C_{2}}{g_{m 2} g_{m 3}}} \\
\mathrm{LP} & \frac{V_{2}}{V_{i n}} & =\frac{1}{1+s \frac{g_{m 2}^{\prime}}{g_{m 2}} \frac{C_{2}}{g_{m 3}}+s^{2} \frac{g_{m 1}^{\prime}}{g_{m 1}} \frac{C_{1} C_{2}}{g_{m 2} g_{m 3}}}
\end{aligned}
$$

Notice that the structure in Fig.6(c) is high pass filter which does not require input signal to be fed through a capacitor. A Gm-C topology with the same property was first reported in [2].

Because of the way the two basic structures are implemented (see equation (4)), the currents $I_{1+}^{\prime}, I_{1-}^{\prime}, I_{2+}^{\prime}$ and $I_{2-}^{\prime}$ can be directly injected into the third transconductor (e.g. Fig. 7) making transistors $M_{1}$ and $M_{2}$ in the doubleoutput structure (Fig. 2(b) and 2(c)) unnecessary. Hence, we conclude only two transistors must be added to a singleoutput transconductor to create a double-output transconductor suitable for implementation of the proposed biquad structures.

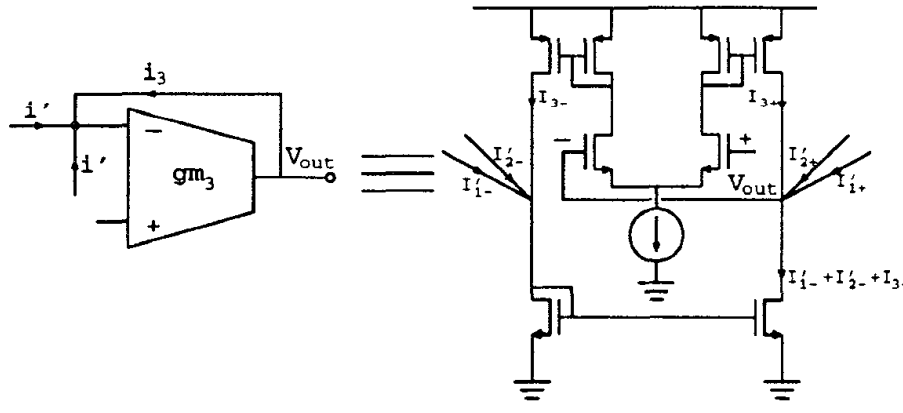

Figure 7: Avoiding the use of additional current mirrors in the double-output transconductors

\section{Effect of Nonidealities on the Performance} of the Proposed Structures

Some of the factors limiting the performance of the proposed structures, and that of any Gm-C filter in general, are the finite input and output impedances and excess phase of the transconductors. The finite output resistance affects mainly the low frequency performance of a lossless integrators. The parasitic capacitances from each node to ground will alter the location of the desired poles and zeros. In addition, any capacitance $C_{\text {out }}$ at output node will contribute a high frequency $\left(g_{m 3} / C_{\text {ovt }}\right)$ pole. It can be shown that the desired poles and zeros of Fig. 4 circuit are not affected by the presence of a nonzero $C_{\text {out }}$. In the next section we show how this property can advantageously be used in an odd-order biquad filter design. Since the integrator loop of the proposed structures consists of only two transconductors the effect of the excess phase on the performance is kept minimum.

\section{RESULTS}

A fifth-order band-limited inverse Gaussian filter using the biquad structure in Fig. 4 was designed and fabricated using the MOSIS 2- $\mu$ Orbit Analog Process. The normalized transfer function of the filter whose block diagram is shown in Fig. 8 is,

$H(s)=\frac{968.38-854.03 s^{2}+1752.25 s^{4}}{945+2268 s+2419.2 s^{2}+1451.5 s^{3}+497.7 s^{4}+79.6 s^{5}}$

The poles and zeros are arranged in such a way that in the normalized frequency range $\omega=0-1.15$ its amplitude response provides equiripple approximation of an inverse Gaussian while having linear (Bessel-Thompson) phase response. This transfer function can be implemented using two biquad structures and a lossy integrator or two Fig. 4 biquads, one of which is loaded with a capacitor (e.g.Fig.). Fig. 9 shows the ideal (as given by (12)) amplitude response and the measured one.

\section{Conclusion}

The derivation of Gm-C biquad topologies which use twooutput transconductors has been presented. The advantages and some practical limitations of these structures have been pointed out. Measured results showing the performance of a 5-th order monolithic inverse Gaussian filter based on the proposed topology have been included.

\section{REFERENCES}

[1] R. L. Geiger and E. Sánchez-Sinencio, "Active filter design using operational transconductance amplifiers; A tutorial," IEEE Circrit and Devices Mag., vol. 1, pp. 20-32, March 1985.

[2] E. Sanchez-Sinencio, R. L. Geiger and H. Nevarez-Lozano, "Generation of continuous-time two integrator loop OTA filter structures," IEEE Transactions on Circuits and Systems, vol. 35, no. 8, pp. 936-945, August 1988.

[3] Chun-Ming Chang and Pang-Chia Chen, "Universal active filter with current gain using OTAs," International Journal of Electronics, vol. 71, no. 5, pp. 805-8, Nov. 1991.

[4] R. Nawrocki and U. Klein, "New OTA-capacitor realization of a universal biquad," Electronics Letters, vol. 22, no. 1, pp. 50-1, Jan. 1986. 

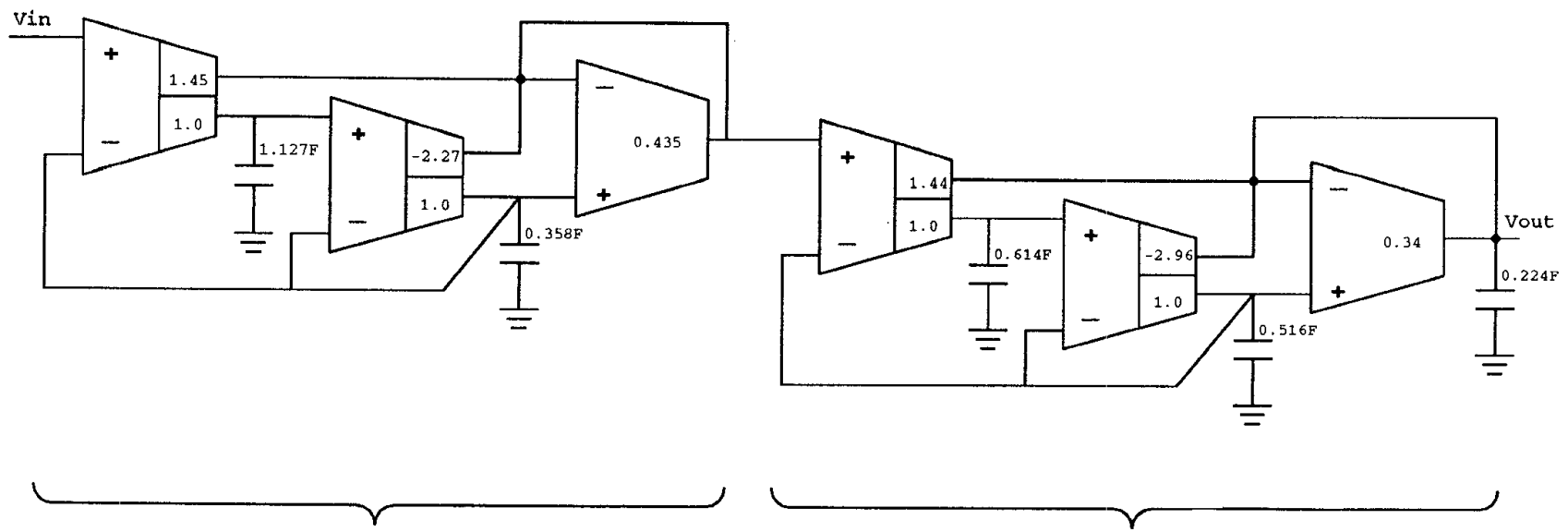

$$
\frac{1+1.891 s+1.346 s^{2}}{1+1.127 s+0.404 s^{2}}
$$$$
\frac{1-1.891 s+1.346 s^{2}}{1+0.615 s+0.317 s^{2}} \frac{1}{1+0.658 s}
$$

Figure 8: Gm-C implementation of band-limited inverse Gaussian

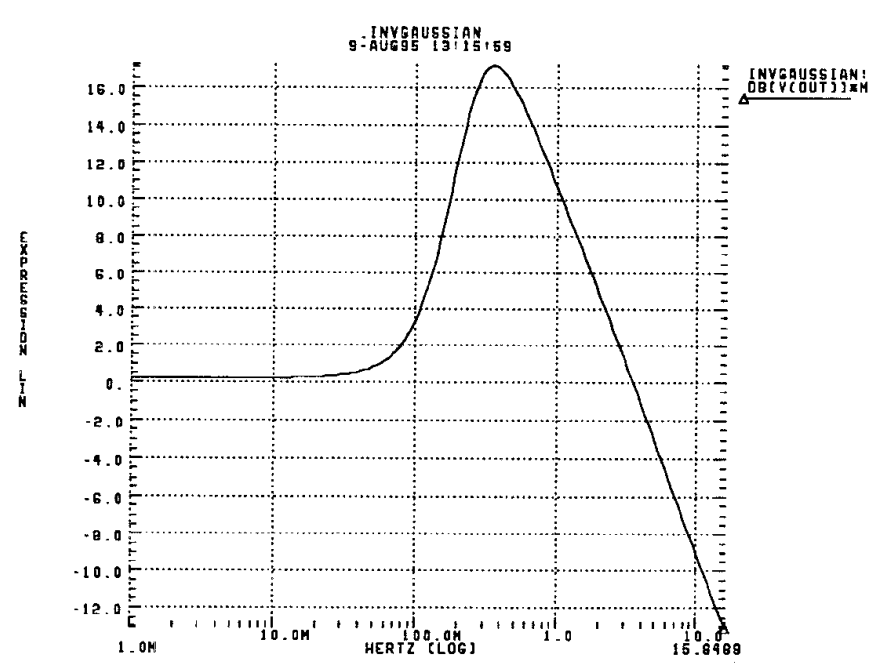

(a)

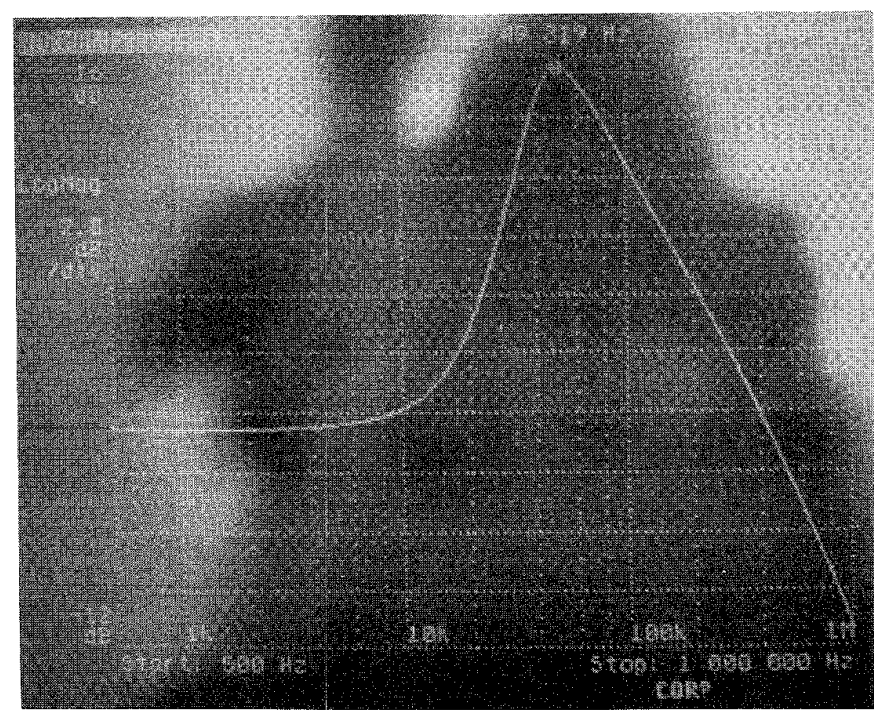

(b)

Figure 9: (a) Ideal Amplitude Response; (b) Measured Amplitude Response 